\title{
Dark Matter Through the Quark Vector Current Portal
}

\author{
Dillon Berger and Arvind Rajaraman \\ Department of Physics and Astronomy, University of California, Irvine, CA 92697, USA \\ Jason Kumar \\ Department of Physics and Astronomy, University of Hawai'i, Honolulu, HI 96822, USA
}

\begin{abstract}
We consider models of light dark matter coupled to quarks through a vector current interaction. For low energies, these models must be treated through the effective couplings to mesons, which are implemented here through the chiral Lagrangian. We find the rates of dark matter annihilation and decay to the light mesons, and find the expected photon spectrum from the decay of the hadrons. We compare to current and future observations, and show that there is a significant discovery reach for these models.
\end{abstract}




\section{INTRODUCTION}

Recently, there has been significant interest in models of dark matter (DM) in which the dark matter particle has a mass $m_{X} \lesssim \mathcal{O}(\mathrm{GeV})$. These models can evade the tight constraints on dark matter placed by direct detection experiments, since these experiments typically lose sensitivity at low mass. If dark matter with $m_{X} \lesssim \mathcal{O}(\mathrm{GeV})$ annihilates or decays in the cosmos, the photons produced will tend to lie in the current "MeV-gap" in observational sensitivity, but a variety of new instruments (such as e-ASTROGAM [1], AMEGO [2] and APT [3]) are being developed to fill this gap. Such instruments would be well-positioned for indirect detection searches for MeV-range dark matter.

There has been particular recent interest in MeV-range dark matter which couples largely to light quarks 4 -10. The reason is because the hadronic final states which can be produced at such small center-of-mass energies are largely constrained by kinematics and symmetry. Moreover, several of the accessible hadrons, such as $\pi^{0}$ and $\eta$, produce striking photon signals when they decay. This scenario is thus particularly appealing from the point of view of indirect detection.

Recent work has considered the case where dark matter couples to either scalar, pseudoscalar, or axial-vector quark bilinears [10]. But if the dark matter couples to a quark vector current, then the leading accessible final state (at low center-of-mass energy) is $\pi^{+} \pi^{-}$, whose decays produce few photons, making this case difficult to probe. In this work, we revisit this case at slightly higher center-of-mass energy $(\sqrt{s} \gtrsim 1 \mathrm{GeV})$, where new final states are allowed. We determine the photon spectrum which will be produced for a variety of choices of the flavor structure of DM-Standard Model (SM) interactions, and determine the sensitivity of proposed experiments.

We assume that electroweak couplings are only relevant for the decays of hadrons produced by dark matter annihilation/decay. As described in 10, (see also 11]), DM-SM interactions can then be understood using chiral perturbation theory, where dark matter is introduced as a spurion which breaks Standard Model flavor symmetries. We will be interested in the case where dark matter appears as a vector spurion. The chiral Lagrangian used for the analysis in [10] only involved the pseudoscalar meson octet. But since this work will consider a higher mass range, we will find that we must also introduce the vector meson octet, following the approach of [13]. We will find that the dominant photon signal arises from the production of $\pi^{0}$, either directly or from the decays of $K_{L}, K_{S}, K^{ \pm}, \rho$ or $\omega$.

The plan of this paper is as follows. In Section II, we will describe the application of chiral perturbation theory to the interaction of low-mass dark matter with quark vector currents. In Section III, we will describe the generation of the photon spectrum through primary and secondary decays of the hadronic final state particles. In Section IV, we will present the sensitivities which can be expected from proposed experiments. We conclude with a discussion of our results.

\section{THE APPLICATION OF CHIRAL PERTURBATION THEORY TO DARK MATTER INTERACTIONS WITH VECTOR CURRENTS}

We will consider two models in which dark matter either decays or annihilates through a coupling to a vector quark current. In the first model of dark matter decay, a single spin-1 dark matter particle $\left(X_{\mu}\right)$ couples to the vector quark current and can decay to Standard Model particles. For this model,

$$
\mathcal{L}_{\text {int }}=g \sum_{q} \alpha_{q} X^{\mu} \bar{q} \gamma_{\mu} q,
$$

In the second model, we take dark matter to be a Dirac fermion $(\chi)$ which couples to quarks through a vector-vector interaction. In this model we take

$$
\mathcal{L}_{i n t}=\sum_{q} \frac{\alpha_{q}}{\Lambda^{2}} \bar{\chi} \gamma^{\mu} \chi \bar{q} \gamma_{\mu} q
$$

For the application to chiral perturbation theory, it is useful to consider these interactions as couplings of the quark vector current to a spurion $v_{q}^{\mu}$ where,

$$
v_{q}^{\mu}=g \alpha_{q} X^{\mu}, \text { or } \frac{\alpha_{q}}{\Lambda^{2}} \bar{\chi} \gamma^{\mu} \chi
$$

Note that this interaction generically breaks the $S U(3)_{L} \times S U(3)_{R}$ flavor symmetries of low-energy QCD.

We will take the mass of the dark matter to be in the $\mathrm{GeV}$ range; we shall be more specific shortly. In this range the decay/annihilation products cannot be treated as weakly coupled propagating quarks. We assume that the dominant final states produced by low-mass dark matter interactions with quarks are hadronic, and that primary interactions which scale as $\alpha_{E M}$ or $s G_{F}$ are negligible. Since the coupling of dark matter to light hadrons is governed by QCD and the dark matter-quark current contact interaction, we can directly express the coupling of dark matter to light mesons using a chiral Lagrangian in which dark matter appears as a spurion which breaks Standard Model flavor symmetries. 
This approach was followed in [10, under the assumption that $\sqrt{s} \lesssim 2 m_{K^{ \pm}}$. In this case, the only accessible hadrons are $\pi^{0}, \pi^{ \pm}$and $\eta$, and one can describe DM-SM interactions using a chiral Lagrangian involving only the spurions and the pseudoscalar meson octet. But in the case in which dark matter only interacted with quark vector currents, it was found that the only accessible two-body final state was $\pi^{+} \pi^{-}$. Since the decays of charged pions produce very few photons, this channel is not useful for the purpose of indirect detection with gamma-ray telescopes.

We now consider the case in which the energy reach is extended to $\sqrt{s} \lesssim 1.15 \mathrm{GeV}$; if we assume that the mass of the lightest glueball state is approximately $1 \mathrm{GeV}$, then this upper cutoff represents the energy scale at which glueball states can be produced along with neutral pions. A treatment of glueball emission is beyond the scope of this work, and although the results we find may be qualitatively correct even for $\sqrt{s} \gtrsim 1.15 \mathrm{GeV}$, we will not study this mass range further.

The amplitude for producing any of the allowed final states from a dark matter initial state can be calculated using the chiral Lagrangian (these states are restricted by symmetry considerations; see Appendix A). We first write the chiral Lagrangian for the pseudoscalar meson octet to lowest order in the $p^{2}$ expansion. As the flavor symmetries of low-energy QCD are broken by the insertion of the $v_{q}^{\mu}$ in the fundamental Lagrangian through eq. 1 . the $v_{q}^{\mu}$ must also appear in the chiral Lagrangian as spurions which break the flavor symmetries. The form of these interactions is determined at this order by symmetry considerations, and chiral Lagrangian can be expressed (see [12]) as

$$
\mathcal{L}_{\Phi}=\frac{F^{2}}{4} \operatorname{Tr}\left[\left(\partial_{\mu} U-i v_{\mu} U+i U v_{\mu}\right)\left(\partial^{\mu} U^{\dagger}+i U^{\dagger} v_{\mu}-i v_{\mu} U^{\dagger}\right)\right]
$$

where

$$
\begin{aligned}
U & \equiv \exp [\imath \sqrt{2} \Phi / F], \\
\Phi & \equiv\left(\begin{array}{ccc}
\frac{\pi^{0}}{\sqrt{2}}+\frac{\eta_{8}}{\sqrt{6}} & \pi^{+} & K^{+} \\
\pi^{-} & -\frac{\pi^{0}}{\sqrt{2}}+\frac{\eta_{8}}{\sqrt{6}} & K^{0} \\
K^{-} & \bar{K}^{0} & -\frac{2 \eta_{8}}{\sqrt{6}}
\end{array}\right) \\
v^{\mu} & \equiv\left(\begin{array}{ccc}
v_{u}^{\mu} & 0 & 0 \\
0 & v_{d}^{\mu} & 0 \\
0 & 0 & v_{s}^{\mu}
\end{array}\right) .
\end{aligned}
$$

The pion decay constant is $F \sim 92 \mathrm{MeV}$. To a good approximation, $\eta_{8}$ can be equated with the physical $\eta$ meson, and we do so henceforth.

It is useful to parameterize the flavor structure of $v_{\mu}$ in terms of its transformation properties under the isospin subgroup of $S U(3)_{L} \times S U(3)_{R}$. In particular, $v_{\mu}$ can be expanded in terms of two isospin singlet components $v^{1 I s}$ and $v^{s 2 I}$, and a component $v^{t I}$ with quantum numbers $I=1, I_{3}=0$ :

$$
\begin{aligned}
v^{s 1 I} & \propto\left(\begin{array}{ccc}
\frac{1}{\sqrt{2}} & 0 & 0 \\
0 & \frac{1}{\sqrt{2}} & 0 \\
0 & 0 & 0
\end{array}\right), \\
v^{s 2 I} & \propto\left(\begin{array}{lll}
0 & 0 & 0 \\
0 & 0 & 0 \\
0 & 0 & 1
\end{array}\right), \\
v^{t I} & \propto\left(\begin{array}{ccc}
\frac{1}{\sqrt{2}} & 0 & 0 \\
0 & -\frac{1}{\sqrt{2}} & 0 \\
0 & 0 & 0
\end{array}\right) .
\end{aligned}
$$

The isospin quantum numbers of the final state should be the same as those of the vector spurion.

The vector spurion only couples to an even number of mesons in $\mathcal{L}_{\Phi}$; since we are only interested in two-body final states, the only relevant terms in eq. 5 are the following contact interactions

$$
\mathcal{L}_{\text {contact }}=\imath\left\{\left(v_{d}^{\mu}-v_{s}^{\mu}\right) \bar{K}^{0} \partial_{\mu} K^{0}+\left(v_{s}^{\mu}-v_{u}^{\mu}\right) K^{+} \partial_{\mu} K^{-}+\left(v_{d}^{\mu}-v_{u}^{\mu}\right) \pi^{+} \partial_{\mu} \pi^{-}-h . c\right\} .
$$

Note that, these interactions respect isospin, U-spin and V-spin, as expected.

Beyond the final states involving two pseudoscalar mesons, the only two-body final states which are kinematically accessible are $\rho \pi$ and $\omega \pi^{0}$. In order to consider these final states, it necessary to include vector mesons in the chiral Lagrangian. For this purpose, we use the results of [13. None of the relevant final states can produced directly by a contact interaction involving the vector meson octet, but they can be produced through a coupling of dark matter to an intermediate vector meson. We thus need the couplings of a vector meson to a vector spurion, and the trilinear couplings involving at least one vector meson. Note that because a neutral vector meson is always produced as an 
intermediate state, the quantum numbers of the intermediate vector meson must be the same as those of the initial state. In particular, the $\omega, \rho^{0}$ and $\phi$ are only produced as intermediate states if the initial state is parameterized by the spurion $v^{s 1 I}, v^{t I}$ or $v^{s 2 I}$, respectively.

The relevant part of the chiral Lagrangian was found in [13, 14, and can be written as

$$
\begin{aligned}
\mathcal{L}_{\Phi_{\mu \nu}}= & -\frac{1}{4} \operatorname{Tr}\left[\left(D^{\mu} \Phi_{\mu \alpha}\right)\left(D_{\nu} \Phi^{\nu \alpha}\right)\right]+\frac{1}{8} m_{V}^{2} \operatorname{Tr}\left[\Phi^{\mu \nu} \Phi_{\mu \nu}\right]+\frac{1}{2} f_{V} \operatorname{Tr}\left[\Phi^{\mu \nu} f_{\mu \nu}^{+}\right]+\frac{i}{2} f_{V} h_{P} \operatorname{tr}\left(U_{\mu} \Phi^{\mu \nu} U_{\nu}\right) \\
& +\frac{i}{8} h_{A} \varepsilon^{\mu \nu \alpha \beta} \operatorname{tr}\left(\left\{\Phi_{\mu \nu},\left(D^{\tau} \Phi_{\tau \alpha}\right)\right\} U_{\beta}\right)+\frac{i}{8} h_{O} \varepsilon^{\mu \nu \alpha \beta} \operatorname{tr}\left(\left[D_{\alpha} \Phi_{\mu \nu}, \Phi_{\tau \beta}\right] U^{\tau}\right)
\end{aligned}
$$

where

$$
\begin{aligned}
\Phi_{\mu \nu} & =\sqrt{2}\left(\begin{array}{ccc}
\frac{\rho_{\mu \nu}}{\sqrt{2}}+\frac{\omega_{\mu \nu}}{\sqrt{2}} & \rho_{\mu \nu}^{+} & K_{\mu \nu}^{*+} \\
\rho_{\mu \nu}^{-} & -\frac{\rho_{\mu \nu}}{\sqrt{2}}+\frac{\omega_{\mu \nu}}{\sqrt{2}} & K_{\mu \nu}^{* 0} \\
K_{\mu \nu}^{*-} & \bar{K}_{\mu \nu}^{* 0} & \phi_{\mu \nu}
\end{array}\right), \\
f_{\mu \nu}^{+} & =\frac{1}{2}\left[e^{\imath \Phi / \sqrt{2} F}\left(\partial_{\mu} v_{\nu}-\partial_{\nu} v_{\mu}\right) e^{-\imath \Phi / \sqrt{2} F}+e^{-\imath \Phi / \sqrt{2} F}\left(\partial_{\mu} v_{\nu}-\partial_{\nu} v_{\mu}\right) e^{\imath \Phi / \sqrt{2} F],}\right. \\
U_{\mu} & =\frac{1}{2} e^{-\imath \Phi / \sqrt{2} F}\left(\partial_{\mu} e^{\imath \sqrt{2} \Phi / F}\right) e^{-\imath \Phi / \sqrt{2} F}-\frac{\imath}{2} e^{-\imath \Phi / \sqrt{2} F} v_{\mu} e^{\imath \Phi / \sqrt{2} F}+\frac{\imath}{2} e^{\imath \Phi / \sqrt{2} F} v_{\mu} e^{-\imath \Phi / \sqrt{2} F}, \\
D_{\alpha} \Phi_{\mu \nu} & =\partial_{\alpha} \Phi_{\mu \nu}+\left[\Gamma_{\alpha}, \Phi_{\mu \nu}\right], \\
\Gamma_{\alpha} & =\frac{1}{2} e^{-\imath \Phi / \sqrt{2} F}\left(\partial_{\mu}-\imath v_{\mu}\right) e^{\imath \Phi / \sqrt{2} F}+\frac{1}{2} e^{\imath \Phi / \sqrt{2} F}\left(\partial_{\mu}-\imath v_{\mu}\right) e^{-\imath \Phi / \sqrt{2} F}, \\
f_{V} & =(140 \pm 14) \mathrm{MeV}, \\
m_{V} \sim & 0.764 \mathrm{GeV}, \\
h_{A} & =2.33 \pm 0.03, \\
h_{P} \sim & 1.75 .
\end{aligned}
$$

The terms that produce the couplings of a single vector meson to the vector spurion are

$$
\mathcal{L}_{\mathrm{vec}}=-\frac{f_{V}}{2}\left(\left(\left(\partial^{\mu} v_{d}^{\nu}-\partial^{\nu} v_{d}^{\mu}\right)-\left(\partial^{\mu} v_{u}^{\nu}-\partial^{\nu} v_{u}^{\mu}\right)\right) \rho_{\mu \nu}^{0}-\left(\left(\partial^{\mu} v_{d}^{\nu}-\partial^{\nu} v_{d}^{\mu}\right)+\left(\partial^{\mu} v_{u}^{\nu}-\partial^{\nu} v_{u}^{\mu}\right)\right) \omega_{\mu \nu}-\sqrt{2}\left(\partial^{\mu} v_{s}^{\nu}-\partial^{\nu} v_{s}^{\mu}\right) \varphi_{\mu \nu}\right)
$$

Finally, the cubic couplings between vector and pseudoscalar meson octets are

$$
\begin{array}{r}
\mathcal{L}_{\text {int }} \supset-\frac{\sqrt{2} h_{A}}{F} \varepsilon^{\mu \nu \alpha \beta}\left[\frac{1}{2} \partial_{\beta} \pi^{0}\left(\rho_{\mu \nu}^{0} \partial^{\tau} \omega_{\tau \alpha}+\omega_{\mu \nu} \partial^{\tau} \rho_{\tau \alpha}^{0}\right)+\partial_{\beta} \pi^{-}\left(\rho_{\mu \nu}^{+} \partial^{\tau} \omega_{\tau \alpha}+\omega_{\mu \nu} \partial^{\tau} \rho_{\tau \alpha}^{+}\right)+c . c\right] \\
-\frac{h_{O}}{\sqrt{2} F} \varepsilon^{\mu \nu \alpha \beta}\left[\left(\partial_{\alpha} \rho_{\mu \nu}^{0}\right)\left(\left(\partial^{\tau} \pi^{0}\right) \omega_{\tau \beta}\right)+\rho_{\tau \beta}^{0}\left(\left(\partial^{\tau} \pi^{0}\right) \partial_{\alpha} \omega_{\mu \nu}\right)+\left(\partial^{\tau} \pi^{-}\left(\omega_{\tau \beta} \partial_{\alpha} \rho^{+}{ }_{\mu \nu}+\rho^{+}{ }_{\tau \beta} \partial_{\alpha} \omega_{\mu \nu}\right)+c . c\right)\right] \\
+i \frac{8 f_{V} h_{P}}{F^{2}}\left[2 \partial_{\mu} \pi^{+} \partial_{\nu} \pi^{-} \rho_{\mu \nu}^{0}+\partial_{\mu} \bar{K}^{0} \partial_{\nu} K^{0}\left(\rho_{\mu \nu}^{0}-\omega_{\mu \nu}-\sqrt{2} \varphi_{\mu \nu}\right)-\partial_{\mu} K^{-} \partial_{\nu} K^{+}\left(\rho_{\mu \nu}^{0}+\omega_{\mu \nu}-\sqrt{2} \varphi_{\mu \nu}\right)\right] .
\end{array}
$$

We have suppressed terms with no neutral vector meson. Interestingly, none of the relevant matrix elements depend on the parameter $h_{O}$. One can easily see from the Feynman rules in Appendix B that, if the center-of-mass momentum of the initial state is purely time-like, then the only non-vanishing polarizations of the intermediate vector meson are mixed time-space. Similarly, in the frame of reference where the outgoing vector meson is at rest, its polarization tensor is also necessarily mixed time-space. It is then clear that all of the terms proportional to $h_{O}$ vanish.

The rates for dark matter to annihilate to primary mesons can now be calculated straightforwardly from this Lagrangian; the details are given in Appendix B. These primary mesons can then decay through multiple decay modes to produce photons. These can be multistep decay processes; for example, the kaon can decay to pions which subsequently decay to photons. In our case, the primary mesons are $\pi^{0}, \pi^{ \pm}, K^{0}, \bar{K}^{0}, K^{ \pm}, \rho, \omega$, and we need to find the photon spectra produced in their decays. The $\pi^{0}$ decays to two photons essentially $100 \%$ of the time, and the $\pi^{ \pm}$ essentially never produce photons. The $\rho^{0}$ decays primarily to charged pions, and hence does not produce photons. But decays of $\rho^{ \pm}$typically produce $\pi^{0}$ as well as $\pi^{ \pm}$, with the subsequent the decays of $\pi^{0}$ yielding photons. For the kaons and the omega, we use the decay modes found in the Particle Data Book [15. We have tabulated in Table I] the important decay modes of the mesons which we have considered along with their branching ratios. Decay modes which are not expected to produce a significant number of photons (e.g. decays involving only charged pions) are not shown.

For the two-body decays, the decay spectrum at rest is fixed by kinematics. The three-body decays are described by a Dalitz plot, which parametrizes the decay kinematics in terms of the final state energies. We use these to find 


\begin{tabular}{|c|c|c|c|c|c|}
\hline \multirow{4}{*}{$K^{+}$} & $\pi^{0} e^{+} \nu$ & $5 \%$ & $K^{S}$ & $\pi^{0} \pi^{0}$ & $30.7 \%$ \\
\hline & $\pi^{0} \mu^{+} \nu$ & $3.4 \%$ & \multirow{2}{*}{$K^{L}$} & $\pi^{0} \pi^{0} \pi^{0}$ & $19.5 \%$ \\
\hline & $\pi^{+} \pi^{0}$ & $20.7 \%$ & & $\pi^{+} \pi^{-} \pi^{0}$ & $12.5 \%$ \\
\hline & $\pi^{+} \pi^{0} \pi^{0}$ & $1.7 \%$ & & $\pi^{+} \pi^{-} \pi^{0}$ & $89 \%$ \\
\hline$\rho^{ \pm}$ & $\pi^{ \pm} \pi^{0}$ & $100 \%$ & & $\pi^{0} \gamma$ & $8 \%$ \\
\hline
\end{tabular}

TABLE I. The relevant decay modes and branching fractions for the mesons produced from dark matter annihilation/decay through the quark vector current portal.

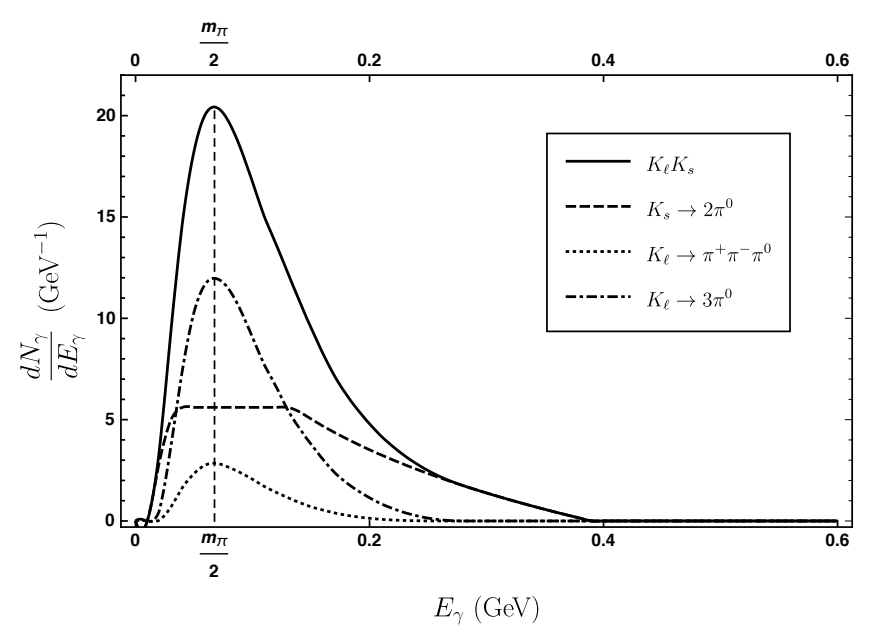

FIG. 1. Photon spectrum from neutral kaon production $\left(K_{L} K_{S}\right)$. The center-of-mass energy has been taken to be 1.14 GeV.

the decay spectrum at rest for each decay mode of each meson. These details are presented in Appendix C. Finally, these mesons are produced and decay with large boosts which also modify the spectrum. The boosting procedure is general, and is described in Appendix D.

For illustrative purposes, we show in Figure 1 the photon spectrum obtained through the production of neutral kaons produced from dark matter annihilation or decay, assuming the $E_{c m}=1.14 \mathrm{GeV}$. The individual spectra have been weighted according to the branching fractions. It is interesting to compare this spectrum to those found in [10, for the case where dark matter coupled to scalar, pseudoscalar or axial-vector quark currents. In those cases, even though the center-of-mass energy was taken to be $\leq 1 \mathrm{GeV}$, the typical photon energy was nearly 4 times larger than found here. The reason is because in the case of a scalar, pseudoscalar or axial-vector spurion, one can produce an $\eta$ in the final state, whose decays yield photons. In the case of a vector spurion, however, almost all photons arise from $\pi^{0}$ decay. As is discussed in Appendix $\mathrm{D}$, the photons produced by $\pi^{0}$ decay yield a typical photon energy which is considerably smaller than that of the photons produced from $\eta$ decay.

\section{COMPARISONS TO OBSERVATIONS}

Following [10], we consider constraints on the obtained photon spectrum from observations of diffuse photon emission, and from future observations of photon emission from a dwarf galaxy (Draco). We will take as a benchmark, an experiment with a fractional $1 \sigma$ energy resolution of $\epsilon=0.3$ and an exposure of $3000 \mathrm{~cm}^{2} \mathrm{yr}$. We also assume that the experimental angular resolution is smaller than the size of Draco $\left(1.3^{\circ}\right)$.

For diffuse emission, we restrict attention to latitudes greater than $>20^{\circ}$. In this region, and in the energy range $0.8 \mathrm{MeV}-1 \mathrm{GeV}$, the isotropic flux observed by COMPTEL and EGRET can be well fit [4, 16, to the function

$$
\frac{d^{2} \Phi^{i s o .}}{d \Omega d E_{o b s .}}=2.74 \times 10^{-3}\left(\frac{E_{o b s}}{\mathrm{MeV}}\right)^{-2.0} \mathrm{~cm}^{-2} \mathrm{~s}^{-1} \mathrm{sr}^{-1} \mathrm{MeV}^{-1}
$$

The expected number of observed events between the energies $E_{-}$and $E_{+}$is then given by

$$
N_{O}=8.6 \times 10^{4}\left(\frac{\mathrm{MeV}}{E_{-}}-\frac{\mathrm{MeV}}{E_{+}}\right) \frac{\left(I_{\text {exp. }} \Delta \Omega\right)}{\mathrm{cm}^{2} \mathrm{yr} \mathrm{sr}} .
$$


To estimate the constraints on the model from diffuse emission, we consider energy bins set by the resolution through $E_{+}-E_{-}=\epsilon\left(E_{+}+E_{-}\right)$. We impose the condition that within any such energy bin, the number of expected signal events should not exceed the number of expected observed events i.e., we require $N_{S}<N_{O}$. This constitutes a conservative bound; since we assume no knowledge of the background photon spectrum, we conservatively assume that the background could be negligible in any energy bin. While in principle we can scan over all possible energy bins, we note that almost all photons arise from tertiary processes in which the decay of a heavier meson produces a $\pi^{0}$, whose decay in turn produces a pair of photons ${ }^{1}$ As discussed in Appendix D, a signal of this form has a peak at $m_{\pi} / 2$, so we shall center our bin at $E_{0}=m_{\pi} / 2$.

For a dwarf spheroidal galaxy, such as Draco, the background consists of all photons emitted by astrophysical processes as well as dark matter annihilation/decay along the line of sight to the dwarf, but not within the dwarf. This background can be estimated purely from data by considering the observed flux in the direction of the dSph, but slightly off-axis [17 21]). Although one would follow this approach in an actual analysis of data from a future instrument, for our purposes, we can estimate the background flux to be roughly the same as observed diffuse flux given in eq. 12 . If we assume that the number of observed events is the same as the expected number of background events, then a model can be ruled out at $n-\sigma$ confidence level if $N_{S}>n \sqrt{N_{O}}$.

\section{RESULTS}

The differential photon flux from dark matter annihilation or decay is

$$
\frac{d^{2} \Phi}{d \Omega d E_{\gamma}}=\frac{\Xi^{\text {ann.,dec. }}}{4 \pi m_{X}} \bar{J}^{\text {ann.,dec. }} \frac{d N_{\gamma}}{d E_{\gamma}},
$$

where

$$
\begin{aligned}
& \Xi^{a n n .}=\frac{\left\langle\sigma_{A} v\right\rangle}{2 m_{X}}, \\
& \Xi^{\text {dec. }}=\Gamma,
\end{aligned}
$$

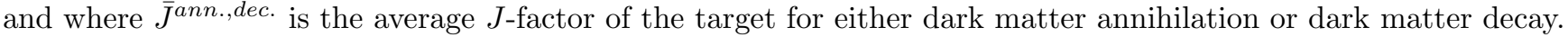

Here, for diffuse emission, the average $J$-factor is 22 ]

$$
\begin{aligned}
& \bar{J}_{\text {dif. }}^{\text {ann. }}=3.5 \times 10^{21} \mathrm{GeV}^{2} \mathrm{~cm}^{-5} \mathrm{sr}^{-1} \\
& \bar{J}_{\text {dif. }}^{\text {dec. }}=1.5 \times 10^{22} \mathrm{GeV} \mathrm{cm}^{-2} \mathrm{sr}^{-1}
\end{aligned}
$$

while for Draco, the average $J$-factor is 23

$$
\begin{aligned}
& \bar{J}_{\text {Draco }}^{\text {ann. }}=6.94 \times 10^{21} \mathrm{GeV}^{2} \mathrm{~cm}^{-5} \mathrm{sr}^{-1}, \\
& \bar{J}_{\text {Draco }}^{\text {dec. }}=5.77 \times 10^{21} \mathrm{GeV} \mathrm{cm}^{-2} \mathrm{sr}^{-1} .
\end{aligned}
$$

We account for instrumental energy resolution by convolving the injected photon spectrum with a Gaussian smearing function

$$
R_{\epsilon}\left(E_{o b s .}, E_{\gamma}\right)=\frac{1}{\sqrt{2 \pi} \epsilon E_{\gamma}} \exp \left(-\frac{\left(E_{o b s .}-E_{\gamma}\right)^{2}}{2 \epsilon^{2} E_{\gamma}^{2}}\right) .
$$

In terms of the the exposure $I_{\text {exp. }}$, and the solid angle $\Delta \Omega$, the number of events expected within the energy window $E_{-} \leq E_{\text {obs. }} \leq E_{+}$is

$$
N_{S}=\frac{\Xi^{\text {ann.,dec. }}}{4 \pi m_{X}} \bar{J}^{\text {ann.,dec. }}\left(I_{\text {exp. }} \Delta \Omega\right) \int_{E_{-}}^{E_{+}} d E_{o b s .} \int_{0}^{\infty} d E_{\gamma} \frac{d N_{\gamma}}{d E_{\gamma}} R_{\epsilon}\left(E_{o b s .}, E_{\gamma}\right)
$$

We now consider the two models described by equations (1) and 2). In each case, we find the photon spectrum from decay/ annihilations and impose bounds from observations. We consider two classes of models. In the first class we assume isospin is a good symmetry, and we set $\alpha_{u}=\alpha_{d}$. In the second class we set $\alpha_{s}=0$.

Bounds on the parameter space of the first class of models are shown in Figure 2, Bounds on the parameter space of the second class of models are shown in Figure 3 . In each case, we have normalized the couplings to have $g=10^{-24}$ in equation (1), and $\Lambda=100 \mathrm{GeV}$ in equation (2). In all cases we have set the center-of-mass energy to be

\footnotetext{
1 Note, there will also be a small number of secondary photons produced through the process $\omega \rightarrow \pi^{0} \gamma$. But since the branching fraction for this process is small, and the resulting feature is in any case not sharp, there is little reason to consider it further.
} 

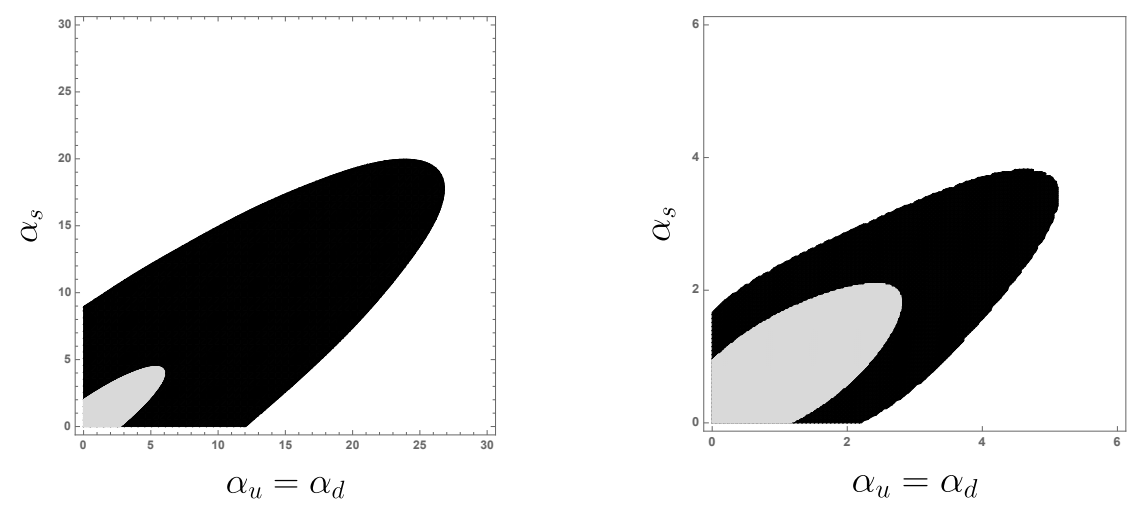

FIG. 2. Bounds on the parameter space from annihilation (left panel, $\Lambda=100 \mathrm{GeV}$ ) and decay (right panel, $g=10^{-24}$ ) on theories with $\alpha_{u}=\alpha_{d}$, assuming $E_{c m}=1.14 \mathrm{GeV}$. The region in black is allowed by constraints on diffuse emission, while the grey region demarcates the sensitivity of a search for emission from Draco.
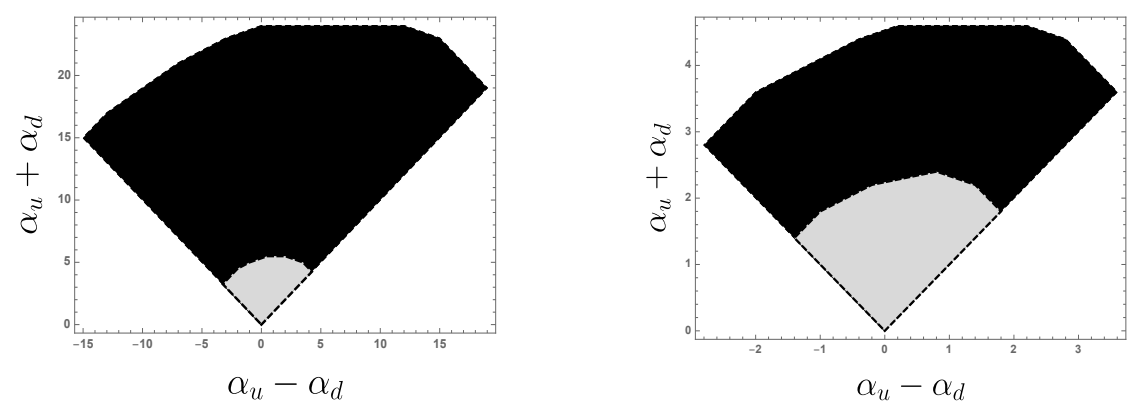

FIG. 3. Bounds on the parameter space from annihilation (left panel, $\Lambda=100 \mathrm{GeV}$ ) and decay (right panel, $g=10^{-24}$ ) on theories with $\alpha_{s}=0$, assuming $E_{c m}=1.14 \mathrm{GeV}$. The region in black is allowed by constraints on diffuse emission, while the grey region demarcates the sensitivity of a search for emission from Draco.

$E_{c m}=1.14 \mathrm{GeV}$. Because the energy range between the $\rho \pi$ threshold $(\sim 0.91 \mathrm{GeV})$ and our upper cutoff $(1.15 \mathrm{GeV})$ is relatively narrow, these constraints do not change dramatically as we change the center-of-mass energy. But there is one feature worthy of note; if $E_{c m} \sim 1.02 \mathrm{GeV}$ and if dark matter couples to a strange quark vector current, then dark matter can annihilate through an intermediate $\phi$ near resonance. In this case, the constraints on $\alpha_{s}$ which could be obtained from indirect detection would improve dramatically.

Note that constraints from Draco are tighter than those from diffuse emission, but more so in the case of dark matter annihilation than in the case of decay. This is what one would expect, as the annihilation rate scales as the square of the density, whereas the decay rate scales only with the density.

Note also that, if the dark matter coupling does not break isospin, then the constraints on the couplings are weakest if $\alpha_{u}=\alpha_{d}=\alpha_{s}$. This can easily be understood from equation (7); if all light quark couplings are identical, the dark matter coupling to the pseudoscalar meson octet vanishes. In this limit, the only remaining channels which produce photons are $\omega \pi^{0}$ and $\rho \pi$. The constraints on dark matter decay (annihilation) obtained from diffuse emission in this limit correspond to $\tau \sim \mathcal{O}\left(10^{25}\right) \mathrm{s}\left(\left\langle\sigma_{A} v\right\rangle \sim \mathcal{O}\left(10^{-25}\right) \mathrm{cm}^{3} / \mathrm{s}\right)$. A similar sensitivity to dark matter decay (annihilation) in Draco could yield bounds of $\tau \sim \mathcal{O}\left(10^{26}\right) \mathrm{s}\left(\left\langle\sigma_{A} v\right\rangle \sim \mathcal{O}\left(10^{-26}\right) \mathrm{cm}^{3} / \mathrm{s}\right)$. The sensitivity to dark matter decay thus exceeds that obtainable by Planck [24] (from the effect of late-time energy injection on the CMB) by about an order of magnitude. But bounds from Planck are much tighter in the case of dark matter annihilation, and exceed the sensitivity we would obtain from a search for emission from Draco by about two orders of magnitude. But if more nearby dwarf spheroidal galaxies are discovered, then one might hope that a stacked analysis of all dwarfs could provide competitive sensitivity.

For the isotropic flux, we have used the best fit to data from COMPTEL and EGRET. But future instruments, with larger exposure and better angular resolution, may be able to identify more point sources. In this case, masking these point sources could result in a significant reduction of the remaining isotropic flux, and the sensitivity of this analysis would improve accordingly.

Finally, we note that direct detection experiments and LHC mono-anything searches are completely unconstraining for models of dark matter decay. As noted in [10, LHC searches are also do not constrain models of dark matter annihilation if the mass scale of the particle mediating the DM-quark interaction is $\mathcal{O}(\mathrm{GeV})$. But for $m_{\chi} \sim 500 \mathrm{MeV}$, the upper bound on spin-independent (SI) dark matter-nucleon scattering found by CRESST [25] is $\sigma_{\mathrm{SI}} \sim \mathcal{O}\left(10^{-1}\right) \mathrm{pb}$. 
For a scenario of dark matter annihilation through coupling to the quark vector portal, DM-nucleon scattering would be spin and velocity-independent. If we set $\alpha_{u}=\alpha_{d} \sim 3, \alpha_{s}=0$ (the limit of sensitivity for a search for emission from Draco), one would find $\sigma_{\mathrm{SI}} \sim \mathcal{O}(10) \mathrm{pb}$, about two orders of magnitude above current bounds from CRESST. However, CRESST's sensitivity is greatly reduced for the case of isospin-violating dark matter [26 28] $\left(\alpha_{u}=-\alpha_{d}, \alpha_{s}=0\right)$, and is essentially unconstraining for the parameter space of interest here. Similarly, if dark matter only couples to the strange quark vector current $\left(\alpha_{u}=\alpha_{d}=0\right)$, then the SI scattering cross section vanishes, and there are no meaningful constraints from direct detection.

\section{CONCLUSIONS}

In conclusion, we have considered dark matter coupled to Standard Model quarks through vector currents of the form $\bar{q} \gamma^{\mu} q$. We have utilized the chiral Lagrangian to obtain couplings of the dark matter to mesons, and found the photon spectrum from dark matter decay (if the dark matter itself is a vector) or from annihilation (where it is a Dirac fermion).

We have found that current observations of the diffuse photon spectrum already can be used to constrain the parameter space of these models. Future observations of dwarf spheroidal galaxies will significantly improve these bounds or may discover these models. In the case of dark matter decay, these constraints exceed those obtainable from Planck by about an order of magnitude.

There are several ways to extend this analysis. We have considered final states with at most two mesons, and have worked to $\mathcal{O}\left(p^{2}\right)$ in the chiral Lagrangian. Consideration of 4-meson final states, and higher order terms in the chiral Lagrangian momentum expansion, would yield subleading corrections to the photon spectrum which may nevertheless be significant, espeically below the $\rho \pi$ threshold. Similarly, if we consider higher center-of-mass energies, then new final state particles will be accessible, including the $\phi$ and glueball states. We hope to return to this in future work.

We have found that if dark matter couples to a vector quark current, then the typical photon energy is roughly a factor of 4 smaller than in the scenarios considered in [10], wherein dark matter coupled to scalar, pseudoscalar or axial-vector currents. This result is largely independent of the center-of-mass energy of the process, but is instead dictated by the Lorentz and flavor structure of the dark matter-quark current coupling, which determines whether or not an $\eta$ can be produced in the final state. It would be interesting to study more quantitatively the ability of future experiments to definitively determine the Lorentz and flavor structure of the DM-quark coupling from the photon distribution.

Finally, we note that, although we have assumed an energy resolution of $30 \%$ and an exposure of $3000 \mathrm{~cm}^{2} \mathrm{yr}$, upcoming experiments may well exceed these performance benchmarks. Energy resolutions of as low as $10 \%$ may be possible [32, 33, as well as exposures which are up to an order of magnitude larger [32.

\section{ACKNOWLEDGEMENTS}

We are grateful to James H. Buckley, Regina Caputo and Xerxes Tata for useful discussions. J. K. is supported in part by DOE grant de-sc0010504. A. R. is supported in part by NSF Grant PHY-1620638.

\section{Appendix A: Symmetry considerations}

Since we assume that the primary dark matter annihilation/decay process does not involve weak interactions, we will find that the hadronic final state has vanishing strangeness, and has the same parity, charge conjugation and angular momentum quantum numbers as the initial state. We also, for simplicity, focus only on primary annihilation/decay processes which produce at most two mesons. As such, the only kinematically accessible neutral two-body final states with vanishing net strangeness are $\pi \pi, \eta \eta, \eta \pi^{0}, \rho \pi, \omega \pi^{0}, K^{+} K^{-}, K^{0} \bar{K}^{0}$.

The quantum numbers of the Standard Model final state can be determined in general from the quantum numbers of the dark matter initial state (for example, see 29]). If dark matter couples to a vector quark current, the final state necessarily has $J^{P C}=1^{--}$. As such, the $\pi^{0} \pi^{0}, \eta \pi^{0}, \eta \eta, K_{L} K_{L}$ and $K_{S} K_{S}$ final states are forbidden. As a result, the only final states which we need consider are $\pi^{+} \pi^{-}, K^{+} K^{-}, K^{L} K^{S}, \rho \pi$, and $\omega \pi^{0}$. Since all of the mesons in question have odd intrinsic parity, each state transforms under parity as $(-1)^{L}$, and must thus have orbital angular momentum $L=o d d$. From the symmetry of the wavefunction, we then see that the $\pi^{+} \pi^{-}$state must have isospin $I=1, I_{3}=0$. 
Similarly, we can classify the two-kaon final states in terms of their isospin quantum numbers:

$$
\begin{array}{ll}
(K K)_{s}=\frac{1}{\sqrt{2}}\left(\left|K^{+} K^{-}\right\rangle+\left|K_{L} K_{S}\right\rangle\right) & I=0, \\
(K K)_{t}=\frac{1}{\sqrt{2}}\left(\left|K^{+} K^{-}\right\rangle-\left|K_{L} K_{S}\right\rangle\right) & I=1, I_{3}=0 .
\end{array}
$$

Note, the choice of which relative sign corresponds to the singlet or triplet state is a convention, which depends on the normalizations of the $\left|K^{+} K^{-}\right\rangle$and $\left|K_{L} K_{S}\right\rangle$ states. The $\omega \pi^{0}$ state is necessarily $I=1, I_{3}=0$, while the $\rho \pi$ state is $I=0,1$ or $2, I_{3}=0$. But since the isospin quantum numbers of the final state should be the same as those of the quark current to which the vector spurion couples, we should only be only be able to produce $\rho \pi$ states with $I=0$ or 1 ; the $I=2 \rho \pi$ state should be inaccessible.

Thus, we essentially have six final states, two of which have $I=I_{3}=0\left((K K)_{s}\right.$ and $\left.(\rho \pi)_{s}\right)$, and four of which have $I=1, I_{3}=0\left((\pi \pi)_{t},(K K)_{t},(\rho \pi)_{t}\right.$ and $\left.(\omega \pi)_{t}\right)$. Note that the $(\pi \pi)_{t}$ state produces no photons, while the $(K K)_{s}$ and $(K K)_{t}$ states produce identical photon spectra.

Isospin is an $S U(2)$ subgroup of the QCD flavor symmetries which relates $u$ - and $d$-quarks. But similarly, there are $S U(2)$ subgroups which related $d$ - and $s$-quarks (U-spin) and $u$ - and $s$-quarks ( $\mathrm{V}$-spin). We thus find that the $K^{+} K^{-}$state must have V-spin $I^{V}=1, I_{3}^{V}=0$, while the states $(1 / \sqrt{2})\left(\left|\pi^{+} \pi^{-}\right\rangle \pm\left|K_{L} K_{S}\right\rangle\right)$ have $I^{V}=I_{3}^{V}=0$ and $I^{V}=1, I_{3}^{V}=0$, respectively. Similarly, the $K^{L} K^{S}$ state must have U-spin $I^{U}=1, I_{3}^{U}=0$, while the states $(1 / \sqrt{2})\left(\left|\pi^{+} \pi^{-}\right\rangle \pm\left|K^{+} K^{-}\right\rangle\right)$have $I^{U}=I_{3}^{U}=0$ and $I^{U}=1, I_{3}^{U}=0$, respectively. Note that U-spin and V-spin are not useful for classifying the $\rho \pi$ and $\omega \pi^{0}$ final states, since the $\rho$ and $\omega$ transform into kinematically inaccessible states under U-spin and V-spin; essentially, the $\rho$ and $\omega$ are necessarily close enough to threshold that the strange quark mass cannot be taken as negligible.

\section{Appendix B: Feynman Rules}

We here collect the relevant Feynman rules. For all rules below, all momenta on the left are entering the vertex, and those on the right are exiting.
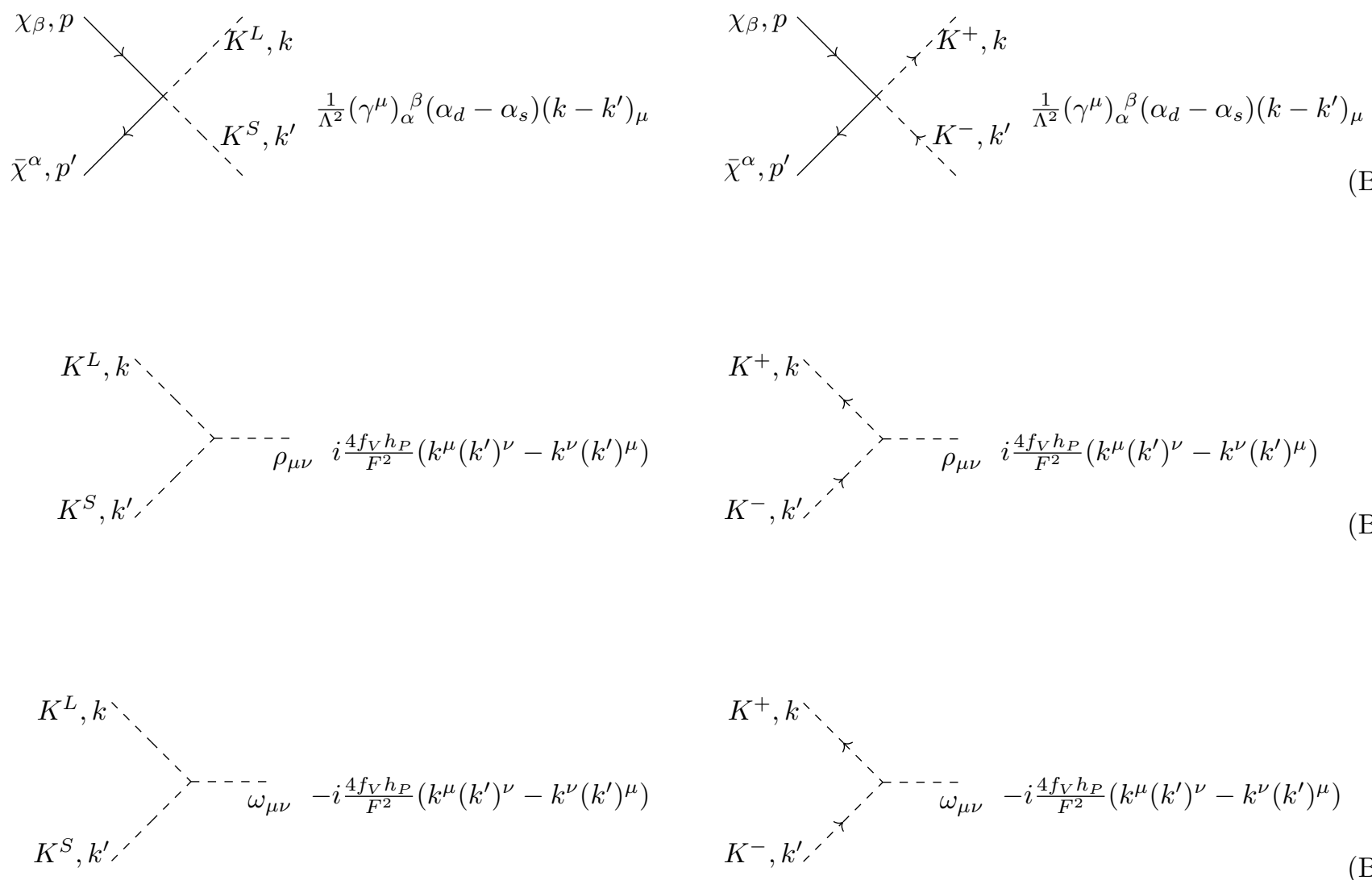

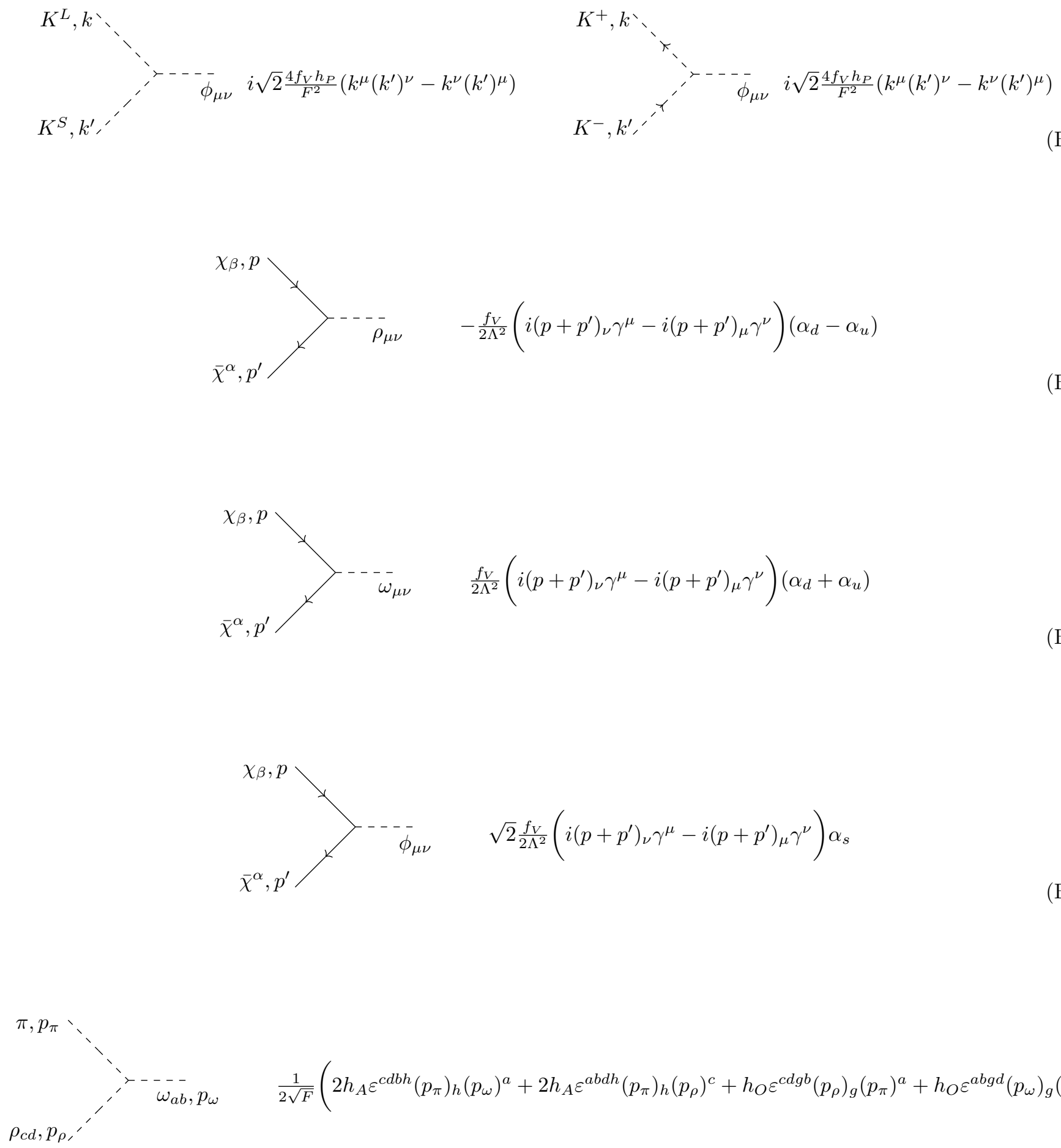

$$
\frac{1}{2 \sqrt{F}}\left(2 h_{A} \varepsilon^{c d b h}\left(p_{\pi}\right)_{h}\left(p_{\omega}\right)^{a}+2 h_{A} \varepsilon^{a b d h}\left(p_{\pi}\right)_{h}\left(p_{\rho}\right)^{c}+h_{O} \varepsilon^{c d g b}\left(p_{\rho}\right)_{g}\left(p_{\pi}\right)^{a}+h_{O} \varepsilon^{a b g d}\left(p_{\omega}\right)_{g}\left(p_{\pi}\right)^{c}\right)
$$

\section{Appendix C: Decay Spectra at Rest}

We begin by finding the decay spectrum at rest for the various mesons. For two-body decays the spectra are set by kinematics. For a decay $A \rightarrow B+C$ the energy spectrum of $B$ is

$$
\frac{d \Gamma}{d E_{B}}=\delta\left(E_{B}-\frac{m_{B}^{2}+m_{A}^{2}-m_{C}^{2}}{2 m_{A}}\right) .
$$


Three body decays must be analyzed in terms of Dalitz plots, which encode the amplitudes as a function of the kinematic variables.

$$
\text { a. } \quad K^{+}\left(p_{K}\right) \rightarrow \bar{\ell}\left(p_{1}\right) \nu\left(p_{2}\right) \pi^{0}\left(p_{3}\right)
$$

The decay to $K^{+}\left(p_{K}\right) \rightarrow \bar{\ell}\left(p_{1}\right) \nu\left(p_{2}\right) \pi^{0}\left(p_{3}\right)$ is controlled by the matrix element $\mathcal{M}=\left(p_{K}+p_{3}\right)^{\mu} \bar{\ell} \gamma_{\mu}\left(1-\gamma_{5}\right) \nu$. The pion energy spectrum is controlled by

$$
\Gamma=\int d m_{12}^{2} d m_{23}^{2} \sum_{\text {spins }}|\mathcal{M}|^{2}
$$

We then find that

$$
\begin{aligned}
\frac{d \Gamma}{d m_{12}^{2} \propto \int d m_{23}^{2}|\mathcal{M}|^{2} \propto} & -8 m_{\ell}^{2}\left(m_{\pi}^{2}+m_{\ell}^{2}-m_{12}^{2}\right)-8 m_{K}^{2} m_{\pi}^{2}\left(\left(m_{23}^{2}\right)_{\max }-\left(m_{23}^{2}\right)_{\min }\right) \\
& +4\left(m_{K}^{2}+2 m_{\ell}^{2}+m_{\pi}^{2}-m_{12}^{2}\right)\left(\left(m_{23}^{4}\right)_{\text {max }}-\left(m_{23}^{4}\right)_{\text {min }}\right) \\
& -\frac{8}{3}\left(\left(m_{23}^{6}\right)_{\max }-\left(m_{23}^{6}\right)_{\min }\right),
\end{aligned}
$$

where we have defined

$$
\begin{gathered}
E_{2}^{*}=\frac{m_{12}^{2}-m_{1}^{2}+m_{2}^{2}}{2 m_{12}} \quad E_{3}^{*}=\frac{M^{2}-m_{12}^{2}-m_{3}^{2}}{2 m_{12}}, \\
m_{i j}^{2}=\left(p_{i}-p_{j}\right)^{2}, \\
\left(m_{23}^{2}\right)_{\max }=\left(E_{2}^{*}+E_{3}^{*}\right)^{2}-\left(\sqrt{\left(E_{2}^{*}\right)^{2}-m_{2}^{2}}-\sqrt{\left(E_{3}^{*}\right)^{2}-m_{3}^{2}}\right)^{2}, \\
\left(m_{23}^{2}\right)_{\min }=\left(E_{2}^{*}+E_{3}^{*}\right)^{2}-\left(\sqrt{\left(E_{2}^{*}\right)^{2}-m_{2}^{2}}+\sqrt{\left(E_{3}^{*}\right)^{2}-m_{3}^{2}}\right)^{2} .
\end{gathered}
$$

$$
\text { b. } \quad K^{+}\left(p_{K}\right) \rightarrow \pi^{0}\left(p_{1}\right) \pi^{0}\left(p_{2}\right) \pi^{+}\left(p_{3}\right)
$$

The decay $K^{+}\left(p_{K}\right) \rightarrow \pi^{0}\left(p_{1}\right) \pi^{0}\left(p_{2}\right) \pi^{+}\left(p_{3}\right)$ can be expressed in terms of the invariant amplitude $\mathcal{A}$ [15]

$$
\begin{aligned}
|\mathcal{A}|^{2} & =\frac{d \Gamma}{d s_{3} d s_{2}} \propto 1+g \frac{\left(s_{3}-s_{0}\right)}{m_{+}^{2}}+h\left(\frac{\left(s_{3}-s_{0}\right)}{m_{+}^{2}}\right)^{2}+k\left(\frac{s_{2}-s_{1}}{m_{+}^{2}}\right)^{2}, \\
& =1+g \frac{\left(s_{3}-s_{0}\right)}{m_{+}^{2}}+h\left(\frac{\left(s_{3}-s_{0}\right)}{m_{+}^{2}}\right)^{2}+k\left(\frac{2 s_{2}+s_{3}-3 s_{0}}{m_{+}^{2}}\right)^{2},
\end{aligned}
$$

where

$$
\begin{array}{r}
s_{i}=\left(p_{K}-p_{i}\right)^{2} \quad s_{0}=\frac{s_{1}+s_{2}+s_{3}}{3}=\frac{1}{3}\left(m_{K}^{2}+m_{1}^{2}+m_{2}^{2}+m_{3}^{2}\right), \\
g=0.626 \quad h=0.052 \quad k=0.0054 .
\end{array}
$$

The pion energy distribution is then

$$
\frac{d \Gamma}{d E_{2}}=-2 m_{K} \frac{d \Gamma}{d s_{2}} \propto \int d s_{3}|\mathcal{A}|^{2}
$$

We then find that the energy distribution of $\pi_{0}$ in decays of $K^{+} \rightarrow \pi^{0} \pi^{0} \pi^{+}$at rest is (here $E_{2}$ is the neutral pion energy)

$$
\frac{d \Gamma}{d E_{2}}=\mathcal{N}_{K^{+} \rightarrow \pi^{+} \pi^{0} \pi^{0} \sigma\left(s_{2}\right)}\left(\alpha_{0}++\alpha s_{2}+\frac{\alpha_{1}}{s_{2}}+\frac{\alpha_{2}}{s_{2}^{2}}+\frac{\alpha_{3}}{s_{3}^{3}}\right)
$$

where

$$
\sigma\left(s_{2}\right)=\frac{\sqrt{\left[-2 m_{+}^{2}\left(m_{\pi}^{2}+s_{2}\right)+\left(m_{\pi}^{2}-s_{2}\right)^{2}+m_{+}^{4}\right]\left[-2 m_{\pi}^{2}\left(m_{K_{+}}^{2}+s_{2}\right)+\left(m_{K_{+}}^{2}-s_{2}\right)^{2}+m_{\pi}^{4}\right]}}{2 m_{+}^{2}}
$$


and

$$
\left\{\begin{array}{l}
\alpha_{0}=-g-\frac{2}{3} h\left(1+\frac{m_{K_{+}}^{2}+2 m_{\pi}^{2}}{m_{+}^{2}}\right), \\
\alpha=\frac{2}{3 m_{+}^{2}} h, \\
\alpha_{1}=2 m_{+}^{2}+\frac{g}{3}\left(m_{K_{+}}^{2}+m_{+}^{2}+2 m_{\pi}^{2}\right)+\frac{h}{9}\left(\frac{20 m_{\pi}^{4}+2 m_{K_{+}}^{4}-4 m_{\pi}^{2} m_{K_{+}}^{2}}{m_{+}^{2}}+16 m_{K_{+}}^{2}-4 m_{\pi}^{2}+2 m_{+}^{2}\right), \\
\alpha_{2}=g\left(m_{\pi}^{2} m_{K_{+}}^{2}-m_{+}^{2} m_{K_{+}}^{2}-m_{\pi}^{4}+m_{+}^{2} m_{\pi}^{2}\right)+\frac{2}{3} h\left(\frac{m_{\pi}^{4} m_{K_{+}}^{2}-2 m_{\pi}^{6}}{m_{+}^{2}}+2 m_{\pi}^{2} m_{K_{+}}^{2}-m_{+}^{2} m_{K_{+}}^{2}-m_{K_{+}}^{4}+m_{\pi}^{4}\right), \\
\alpha_{3}=2 h \frac{\left(m_{+}^{2}-m_{\pi}^{2}\right)^{2}\left(m_{\pi}^{2}-m_{K_{+}}^{2}\right)^{2}}{3 m_{+}^{2}} .
\end{array}\right.
$$

We find that the normalization constant by integrating both sides with respect to $d E$ (picking up a factor of $-\frac{1}{2 m_{K_{+}}}$). The normalization constant is given by,

$$
\begin{gathered}
\mathcal{N}_{K^{+} \rightarrow \pi^{+} \pi^{0} \pi^{0}}=-0.00187217(\mathrm{GeV})^{3} . \\
\text { c. } \quad K_{L}\left(p_{K}\right) \rightarrow \pi^{+}\left(p_{1}\right) \pi^{-}\left(p_{2}\right) \pi^{0}\left(p_{3}\right), \pi^{0}\left(p_{1}\right) \pi^{0}\left(p_{2}\right) \pi^{0}\left(p_{3}\right)
\end{gathered}
$$

The decay $K_{L}\left(p_{K}\right) \rightarrow \pi^{+}\left(p_{1}\right) \pi^{-}\left(p_{2}\right) \pi^{0}\left(p_{3}\right)$ can be expressed in terms of the invariant amplitude $\mathcal{A}[15]$

$$
\left|\mathcal{A}\left(s_{1}, s_{2}, s_{3}\right)\right|^{2}=1+g \frac{\left(s_{3}-s_{0}\right)}{m_{\pi^{+}}^{2}}+h\left(\frac{s_{3}-s_{0}}{m_{\pi^{+}}^{2}}\right)^{2}+j \frac{\left(s_{2}-s_{1}\right)}{m_{\pi^{+}}^{2}}+k\left(\frac{s_{2}-s_{1}}{m_{\pi^{+}}^{2}}\right)^{2}+f \frac{\left(s_{2}-s_{1}\right)}{m_{\pi^{+}}^{2}} \frac{\left(s_{3}-s_{0}\right)}{m_{\pi^{+}}^{2}}+\ldots .
$$

Here

$$
\begin{array}{r}
s_{i}=\left(p_{K}-p_{i}\right)^{2} \quad s_{0}=\frac{s_{1}+s_{2}+s_{3}}{3}=\frac{1}{3}\left(m_{K}^{2}+m_{1}^{2}+m_{2}^{2}+m_{3}^{2}\right), \\
g=0.678 \quad h=0.076 \quad j=0.001 \quad f=0.0045 \quad k=0.0099 .
\end{array}
$$

The pion energy distribution is then (here $E_{3}$ is the neutral pion energy)

$$
\frac{d \Gamma}{d E_{3}} \propto \int d s_{2}|\mathcal{A}|^{2}
$$

We then find that the energy distribution of $\pi_{0}$ in decays of $K_{L} \rightarrow \pi^{+} \pi^{-} \pi^{0}$ at rest is proportional to

$$
\begin{aligned}
\frac{d \Gamma}{d E_{3}}= & {\left[1+g \frac{\left(s_{3}-s_{0}\right)}{m_{\pi}^{2}}+h\left(\frac{\left(s_{3}-s_{0}\right)}{m_{\pi}^{2}}\right)^{2}+\left(j+f \frac{\left(s_{3}-s_{0}\right)}{m_{\pi}^{2}}\right) \frac{\left(-3 s_{0}-s_{3}\right)}{m_{\pi}^{2}}+k\left(\frac{\left(-3 s_{0}-s_{3}\right)}{m_{\pi}^{2}}\right)^{2}\right] \sigma } \\
& +\left(j+f \frac{\left(s_{3}-s_{0}\right)}{m_{\pi}^{2}}+2 k \frac{\left(-3 s_{0}-s_{3}\right)}{m_{\pi}^{2}}\right) \sigma\left(3 s_{0}-s\right) \\
& +\frac{4}{3} k \frac{1}{m_{\pi}^{4}} \sigma\left(\frac{3}{4}\left(3 s_{0}-s\right)^{2}+\frac{1}{4} \sigma^{2}\right)
\end{aligned}
$$

where

$$
\sigma=\sqrt{1-\frac{4 M_{\pi}^{2}}{s}}\left(s-\left(M_{K}+M_{\pi}\right)^{2}\right)^{1 / 2}\left(s-\left(M_{K}-M_{\pi}\right)^{2}\right)^{1 / 2} .
$$

For the $K_{L} \rightarrow 3 \pi^{0}$ decay, we have 15

$$
|\mathcal{A}|^{2}=1+h \frac{\left(s_{3}-s_{0}\right)^{2}}{m_{+}^{4}}
$$

where $h=0.59$. The energy distribution may then be found similarly to the calculation for $K_{L} \rightarrow \pi^{+} \pi^{-} \pi^{0}$ above.

$$
\text { d. } \omega \rightarrow \pi^{0}\left(p_{1}\right) \pi^{+}\left(p_{2}\right) \pi^{-}\left(p_{3}\right)
$$

$\omega$ decays to three pions are described by a similar Dalitz plot, which was taken from [30]. 


\section{Appendix D: Boosting the Decay Spectrum}

We now describe the general procedure to obtain the boosted spectrum from the decay spectrum at rest.

We consider a particle of mass $m$ which decays into a number of daughter particles, and we assume that the kinematic distribution of the decay is known in the rest frame of the particle. Our goal is to determine the kinematic distribution in the lab frame, where the parent particle is moving. We take the parent particle to be traveling along the $z$-axis, with an energy $E_{m}$, corresponding to a Lorentz factor $\gamma=E_{m} / m$. We assume that there is no correlation between the direction of the daughter particle's momentum and the direction of the parent particle's boost.

In the CM frame, the four-momentum of one of the daughter particles is $\left(E^{\prime}, p^{\prime} \sin \theta^{\prime}, 0, p^{\prime} \cos \theta^{\prime}\right)$. We are given $d P / d E^{\prime}$ in the CM frame; i.e. the probability of obtaining in the CM frame a given value of the daughter particle's energy. In the lab frame, the four-momentum is $(E, p \sin \theta, 0, p \cos \theta)$. We are looking for $d P / d E$.

For the daughter particle in the lab frame, we have

$$
E=\gamma\left(E^{\prime}+p^{\prime} \beta \cos \theta\right)
$$

For any given $E$, this equation has a solution for $\cos \theta$ if $E^{\prime}$ lies in the range

$$
\gamma(E-p \beta) \leq E^{\prime} \leq \gamma(E+p \beta)
$$

The kinematic distribution of the daughter particle in the laboratory frame is then

$$
\begin{aligned}
\frac{d P(E)}{d E} & =\frac{1}{2} \int d E^{\prime} d \cos \theta \frac{d P\left(E^{\prime}\right)}{d E^{\prime}} \delta\left(E-\gamma\left(E^{\prime}+\beta p^{\prime} \cos \theta\right)\right), \\
& =\frac{1}{2} \int_{E_{1}}^{E_{2}} d E^{\prime} \frac{d P\left(E^{\prime}\right)}{d E^{\prime}} \frac{1}{p^{\prime} \beta \gamma},
\end{aligned}
$$

where $E_{2}=\gamma(E+p \beta)$ and $E_{1}=\gamma(E-p \beta)$. This formula allows us to obtain the boosted spectrum from the decay spectrum at rest.

If we assume that the parent particle itself has a kinematic distribution in the laboratory frame given by $d N_{m} / d E_{m}$, we then find

$$
\frac{d P(E)}{d E}=\frac{1}{2} \int d E_{m} \frac{d N_{m}}{d E_{m}} \int_{E_{1}\left(E_{m}\right)}^{E_{2}\left(E_{m}\right)} d E^{\prime} \frac{d P\left(E^{\prime}\right)}{d E^{\prime}} \frac{1}{p^{\prime} \beta \gamma} .
$$

Moreover, if the daughter particle itself decays isotropically to some tertiary product, one can determine kinematic distribution of this tertiary product by simply repeating the above process, treating the daughter particle now as the parent to the tertiary particle.

We can apply this formalism to the case of the $\pi^{0}$, whose dominant decay is to two photons. In the rest frame, the photons have back-to-back momenta and the distribution is $d P / d E^{\prime}=2 \delta\left(E^{\prime}-\frac{m_{\pi}}{2}\right)$, where the factor of two accounts for the two photons. We then find

$$
\begin{aligned}
\frac{d P}{d E} & =\int_{E \gamma(1-\beta)}^{E \gamma(1+\beta)} d E^{\prime} \delta\left(E^{\prime}-\frac{m_{\pi}}{2}\right) \frac{1}{E^{\prime} \beta \gamma} \\
& =\frac{2}{\sqrt{E_{\pi}^{2}-m_{\pi}^{2}}} \times\left[\theta\left(E-\frac{m_{\pi}}{2} \sqrt{\frac{1-\beta}{1+\beta}}\right) \theta\left(\frac{m_{\pi}}{2} \sqrt{\frac{1+\beta}{1-\beta}}-E\right)\right]
\end{aligned}
$$

This reproduces the usual box distribution.

If the $\pi^{0}$ injection spectrum is given by $d N_{\pi} / d E_{\pi}$, then we may express the photon spectrum as 31 ]

$$
\frac{d N_{\gamma}}{d E_{\gamma}}=\int_{\frac{m_{\pi}}{2}\left(\frac{2 E_{\gamma}}{m_{\pi}}+\frac{m_{\pi}}{2 E_{\gamma}}\right)}^{\infty} d E_{\pi}\left[\frac{d N_{\pi}}{d E_{\pi}} \frac{2}{\sqrt{E_{\pi}^{2}-m_{\pi}^{2}}}\right]
$$

This implies that the photon spectrum is log-symmetric about $m_{\pi} / 2$ with a global maximum at that point. Moreover, the spectrum decreases monotonically as the energy either increases or decreases away from $m_{\pi} / 2$. We see these features in Figure 1 .

The last thing which is needed is $d N_{\pi} / d E_{\pi}$. This can be determined from the procedure described above, treating the $\pi^{0}$ as the daughter produced by the decay of $K_{L}, K_{S}, K^{ \pm}, \rho^{ \pm}$and $\omega$. But there is one subtlety to note. This approach is strictly valid only if there is no correlation between the pion boost and the boost of the parent. This is necessarily true if the parent is spin-0, but need not be true if the parent is a vector meson. But we will assume that this effect is negligible, and ignore it henceforth.

[1] A. De Angelis et al. [e-ASTROGAM Collaboration], arXiv:1711.01265 [astro-ph.HE]. 
[2] R. Caputo et al. [AMEGO Team], PoS ICRC 2017, 910 (2017). doi:10.22323/1.301.0910

[3] J. H. Buckley and APT Collaboration, "The Advanced Pair Telescope (APT) Mission Concept," in AAS/High Energy Astrophysics Division \#10, vol. 10, p. \#37.04. Mar., 2008.

[4] K. K. Boddy and J. Kumar, Phys. Rev. D 92, no. 2, 023533 (2015) doi:10.1103/PhysRevD.92.023533 arXiv:1504.04024 [astro-ph.CO]].

[5] K. K. Boddy and J. Kumar, AIP Conf. Proc. 1743, 020009 (2016) doi:10.1063/1.4953276 arXiv:1509.03333 [astro-ph.CO]].

[6] K. K. Boddy, K. R. Dienes, D. Kim, J. Kumar, J. C. Park and B. Thomas, Phys. Rev. D 94, no. 9, 095027 (2016) doi:10.1103/PhysRevD.94.095027 arXiv:1606.07440 [hep-ph]].

[7] R. Bartels, D. Gaggero and C. Weniger, JCAP 1705, no. 05, 001 (2017) doi:10.1088/1475-7516/2017/05/001 arXiv:1703.02546 [astro-ph.HE]].

[8] O. Cata, A. Ibarra and S. Ingenhutt, JCAP 1711, no. 11, 044 (2017) doi:10.1088/1475-7516/2017/11/044 arXiv:1707.08480 [hep-ph]].

[9] M. Dutra, M. Lindner, S. Profumo, F. S. Queiroz, W. Rodejohann and C. Siqueira, JCAP 1803, 037 (2018) doi:10.1088/1475-7516/2018/03/037 arXiv:1801.05447[hep-ph]].

[10] J. Kumar, arXiv:1808.02579 [hep-ph].

[11] D. Choudhury and D. Sachdeva, arXiv:1903.06049 [hep-ph].

[12] J. Gasser and H. Leutwyler, Nucl. Phys. B 250, 465 (1985);

J. Gasser and H. Leutwyler, Annals Phys. 158, 142 (1984) ;

U. G. Meissner, Rept. Prog. Phys. 56, 903 (1993) hep-ph/9302247;

G. Ecker, Prog. Part. Nucl. Phys. 35, 1 (1995) hep-ph/9501357;

A. Pich, Rept. Prog. Phys. 58, 563 (1995) hep-ph/9502366;

G. Colangelo and G. Isidori, hep-ph/0101264

S. Scherer, Adv. Nucl. Phys. 27, 277 (2003) hep-ph/0210398.

[13] C. Terschlusen, S. Leupold and M. F. M. Lutz, Eur. Phys. J. A 48, 190 (2012) doi:10.1140/epja/i2012-12190-6 arXiv:1204.4125 [hep-ph]].

[14] C. Terschlsen and S. Leupold, Phys. Rev. D 94, no. 1, 014021 (2016) doi:10.1103/PhysRevD.94.014021 arXiv:1603.05524 [hep-ph]].

[15] M. Tanabashi et al. (Particle Data Group), Phys. Rev. D 98, 030001 (2018).

[16] A. W. Strong, I. V. Moskalenko and O. Reimer, Astrophys. J. 613, 962 (2004) doi:10.1086/423193 astro-ph/0406254.

[17] A. Geringer-Sameth and S. M. Koushiappas, Phys. Rev. Lett. 107, 241303 (2011) doi:10.1103/PhysRevLett.107.241303 arXiv:1108.2914 [astro-ph.CO]].

[18] M. N. Mazziotta, F. Loparco, F. de Palma and N. Giglietto, Astropart. Phys. 37, 26 (2012) doi:10.1016/j.astropartphys.2012.07.005 arXiv:1203.6731 [astro-ph.IM]].

[19] A. Geringer-Sameth, S. M. Koushiappas and M. G. Walker, Phys. Rev. D 91, no. 8, 083535 (2015) doi:10.1103/PhysRevD.91.083535 arXiv:1410.2242 [astro-ph.CO]].

[20] K. Boddy, J. Kumar, D. Marfatia and P. Sandick, Phys. Rev. D 97, no. 9, 095031 (2018) doi:10.1103/PhysRevD.97.095031 arXiv:1802.03826 [hep-ph]].

[21] A. Albert et al. [HAWC Collaboration], Astrophys. J. 853, no. 2, 154 (2018) doi:10.3847/1538-4357/aaa6d8 arXiv:1706.01277 [astro-ph.HE]].

[22] M. Cirelli et al., JCAP 1103, 051 (2011) Erratum: [JCAP 1210, E01 (2012)] doi:10.1088/1475-7516/2012/10/E01, 10.1088/1475-7516/2011/03/051 arXiv:1012.4515 [hep-ph]].

[23] A. Geringer-Sameth, S. M. Koushiappas and M. Walker, Astrophys. J. 801, no. 2, 74 (2015) doi:10.1088/0004637X/801/2/74 arXiv:1408.0002 [astro-ph.CO]].

[24] N. Aghanim et al. [Planck Collaboration], arXiv:1807.06209 [astro-ph.CO].

[25] H. Kluck et al. [CRESST Collaboration], arXiv:1711.01285 [astro-ph.IM].

[26] S. Chang, J. Liu, A. Pierce, N. Weiner and I. Yavin, JCAP 1008, 018 (2010) doi:10.1088/1475-7516/2010/08/018 arXiv:1004.0697 [hep-ph]].

[27] J. L. Feng, J. Kumar, D. Marfatia and D. Sanford, Phys. Lett. B 703, 124 (2011) doi:10.1016/j.physletb.2011.07.083 arXiv:1102.4331 [hep-ph]].

[28] J. L. Feng, J. Kumar and D. Sanford, Phys. Rev. D 88, no. 1, 015021 (2013) doi:10.1103/PhysRevD.88.015021 arXiv:1306.2315 [hep-ph]].

[29] J. Kumar and D. Marfatia, Phys. Rev. D 88, no. 1, 014035 (2013) doi:10.1103/PhysRevD.88.014035 arXiv:1305.1611 [hep-ph]].

[30] P. Adlarson et al. [WASA-at-COSY Collaboration], Phys. Lett. B 770, 418 (2017) doi:10.1016/j.physletb.2017.03.050 arXiv:1610.02187 [nucl-ex]].

[31] K. K. Boddy, K. R. Dienes, D. Kim, J. Kumar, J. C. Park and B. Thomas, Phys. Rev. D 95, no. 5 , 055024 (2017) doi:10.1103/PhysRevD.95.055024 arXiv:1609.09104 [hep-ph]].

[32] J. H. Buckley, private communications.

[33] AMEGO Collaboration website, https://asd.gsfc.nasa.gov/amego/technical.html. 